\title{
A possible solution for the lack of EHB binaries in globular clusters
}

\author{
Z. Han
}

\author{
National Astronomical Observatories / Yunnan Observatory, the Chinese Academy of Sciences, Kunming 650011, PR China \\ e-mail: zhanwenhan@hotmail.com \\ Received 20 February 2008 / Accepted 27 April 2008
}

ABSTRACT

\begin{abstract}
Context. The binary fraction among extreme horizontal branch (EHB) stars in Galactic globular clusters (GCs) is an order of magnitude lower than the binary fraction among their counterparts, field hot subdwarfs. This casts serious doubt on their formation channels. Aims. In this Letter, I explain the difference between the field and the cluster EHB stars with the binary model of Han et al. (2002, MNRAS, 336, 449; 2003, MNRAS, 341, 669) for the formation of EHB stars.

Methods. With the binary population synthesis code of Han et al. (2002, 2003), I follow the evolution of simple stellar populations resulting from single star bursts (note that Han et al. 2002, 2003, adopted a constant star formation rate over the last 15 Gyr for the production of field EHB stars), and obtain EHB stars at different stellar population ages.

Results. I found that the binary fraction among EHB stars decreases with the stellar population age. The fraction of EHB binaries with orbital periods $P<5 \mathrm{~d}$ is $\sim 2.5 \%$ for a stellar population of $10 \mathrm{Gyr}$ from the standard simulation set.

Conclusions. The binary model of Han et al. $(2002,2003)$ is able to explain the lack of EHB binaries in globular clusters. I also propose that the precise determination of the physical parameters of close EHB binaries in GCs can lead to the strictest constraint on common-envelope ejection efficiency.
\end{abstract}

Key words. stars: horizontal-branch - binaries: close - stars: subdwarfs - globular clusters: general

\section{Introduction}

Extreme horizontal branch (EHB) stars (also known as hot subdwarfs) are generally considered to be helium-core-burning stars with extremely thin hydrogen envelopes $\left(<0.02 M_{\odot}\right)$ (Heber 1986; Saffer et al. 1994). They play an important role in many aspects of astrophysics, e.g., stellar evolution, distance indicators, Galactic structure, and the long-standing problem of farultraviolet excess in early-type galaxies (Kilkenny et al. 1997; Green et al. 1986; Han et al. 2007).

Various scenarios have been proposed to explain the formation of EHB stars (Mengel et al. 1976; Webbink 1984; Tutukov \& Yungelson 1990; Sweigart 1997; D'Cruz et al. 1996; Lee 1994; Bressan et al. 1994; Yi et al. 1997), and most of these scenarios are based on single star evolution (see, however, Mengel et al. 1976) or are meant for single EHB stars (see Webbink 1984; Tutukov \& Yungelson 1990, for the merger of two helium white dwarfs (WDs) to form a single EHB star). Some of the models are also quite ad hoc (see Han et al. 2007, for a review). Observationally, about two-thirds of field EHB stars are in close binaries (Maxted et al. 2001), and this presented a serious challenge to the theory for the formation of EHB stars. Han et al. $(2002,2003)$ proposed an a priori binary model (hereafter HPMM model) for the formation of EHB binaries and single EHB stars. In the model, there are three types of formation channels for EHB stars, involving stable Roche lobe overflow (RLOF) for EHB binaries with long orbital periods, commonenvelope (CE) (Paczyński 1976) ejection for EHB binaries with short orbital periods, and the merger of helium WDs to form single EHB stars. The model explains the main observational characteristics of field EHB stars: in particular, their distributions in the orbital period-minimum companion mass diagram, and in the effective temperature-surface gravity diagram; their distributions of orbital period and mass function; their binary fraction and the fraction of EHB binaries with WD companions; their birth rates; and their space density. The HPMM model is a significant advancement and is widely used in the study of EHB stars (O'Tool et al. 2004).

The majority of field EHB stars are in close binaries, and HPMM model explained the binary fraction. Therefore, it was a great surprise that radial-velocity surveys revealed a remarkable lack of close binary systems in EHB stars in globular clusters (GCs) (Moni Bidin et al. 2006, 2008). This may imply that the formation channels for cluster EHB stars are different from those for field EHB stars. However, by checking the channels in the HPMM model and the model results, Moni Bidin et al. (2008) speculated that a binary fraction-age relation may be responsible for the lack of EHB binaries. Does the relation really exist? If it exists, is it able to explain the lack of EHB binaries in GCs?

In this Letter, I carry out detailed Monte Carlo simulations for the formation of EHB stars in GCs with the binary population synthesis code used for the HPMM model, and obtain the binary fraction-age relation, which can explain the lack of EHB binaries in GCs.

\section{The model}

In the HPMM model, EHB stars are mainly produced from three types of channels: stable RLOF, CE ejection, and the merger of helium WD pairs. For a "primordial" binary system, the primary (initially more massive component) evolves and may fill its Roche lobe when it has a helium core. Given the mass ratio $q$ of the primary to the secondary less than a critical value, the mass transfer is dynamically stable and leads to the formation of a wide EHB binary with a main-sequence (MS) companion if the helium core is ignited later (1st stable RLOF channel). Given the mass ratio over the critical value, a CE is formed, and the CE ejection produces a close EHB binary with a MS 
companion if the helium core is ignited later (1st CE ejection channel). Some of the "primordial" binary systems may evolve to WD binary systems after the first mass transfer phase. In this case, the secondary continues to evolve and may fill its Roche lobe when it has a helium core. If the mass transfer is stable, a wide EHB binary with a WD companion is resulted given that the helium core is ignited later ( 2 nd stable RLOF channel). The mass transfer is very likely to be dynamically unstable, and this results in the formation of a $\mathrm{CE}$, and the $\mathrm{CE}$ ejection produces a close EHB binary with a WD companion if the helium core is ignited later (2nd CE ejection channel). For a WD binary system, if the WD is a helium type and the secondary is on the first giant branch (i.e., containing a helium core) when it fills its Roche lobe, the resultant CE contains helium WD pairs. The ejection of the CE leads to the formation of close helium WD pairs, and the close helium WD pairs may merge due to angular momentum loss of gravitational wave radiation to form single EHB stars (the merger channel).

Binaries with primary's initial mass, $M_{1 \mathrm{i}} \sim 0.95-7 M_{\odot}$ and initial orbital period $P_{\mathrm{i}} \sim 1-1000 \mathrm{~d}$, may produce EHB stars via the channels described above. For the 1st stable RLOF channel, the primary fills its Roche lobe on the Hertzsprung gap or the first giant branch, and the mass transfer strips the primary of its envelope and leaves a naked helium core. This leads to a wide $\mathrm{EHB}+\mathrm{MS}$ binary, if helium is ignited. For a stellar population of age $t<1 \mathrm{Gyr}\left(M_{1 \mathrm{i}} \gtrsim 2 M_{\odot}\right)$, a binary with $P_{\mathrm{i}} \sim$ $1-100 \mathrm{~d}$ can evolve to a wide EHB+MS binary via stable RLOF on the Hertzsprung gap or the first giant branch, and a larger EHB mass corresponds to a small $t$ (a large $M_{1 \mathrm{i}}$ ). For $t>1 \mathrm{Gyr}$ $\left(M_{1 \mathrm{i}} \lesssim 2 M_{\odot}\right)$, only RLOF near the tip of the first giant branch (i.e., with a narrow range of $P_{\mathrm{i}}$, typically $\left.\Delta \log \left(P_{\mathrm{i}} / \mathrm{d}\right) \sim 0.5\right)$ can lead to EHB binaries, as the naked helium core is not ignited if the RLOF is not close to the tip of the first giant branch (see Table 4 of Han et al. 2002). The corresponding orbital period $P_{\mathrm{i}}$ is from $\sim 10 \mathrm{~d}$ to $\sim 1000 \mathrm{~d}$ for a stellar population with age $t$ of 1 Gyr to 15 Gyr. The orbital period and the EHB mass of the resultant EHB binary would be larger for a large $t$. For the 1st CE channel to form an EHB binary, the primary of a binary system needs to fill its Roche lobe while it has a helium core. However, the resultant CE cannot be ejected to form a close EHB binary due to a tight envelope if $t<1 \mathrm{Gyr}\left(M_{1 \mathrm{i}} \gtrsim 2 M_{\odot}\right)$. For $t>1 \mathrm{Gyr}$, the primary of a binary system needs to fill its Roche lobe very close to the tip of the first giant branch (i.e., with a typical range of initial orbital period $\left.\Delta \log \left(P_{\mathrm{i}} / \mathrm{d}\right) \sim 0.1\right)$ (see Fig. 1 of Han et al. 2002), otherwise the helium core cannot be ignited after the $\mathrm{CE}$ ejection. A large $t$ (a small $M_{1 \mathrm{i}}$ ) corresponds to a larger $P_{\mathrm{i}}\left(P_{\mathrm{i}} \sim 100-1000 \mathrm{~d}\right.$ for $\left.t \sim 1-15 \mathrm{Gyr}\right)$ and a larger orbital period $P$ of the resultant EHB binary. This channel produces a close EHB binary with a typical EHB mass of $\sim 0.46 M_{\odot}$. No EHB star can form from the 2 nd stable RLOF channel in the HPMM model, as the mass ratio of the secondary (with an appropriate helium core) to its WD primary is too large and the RLOF is not stable. For the second CE channel to form an EHB star, the primary of a binary system first needs to experience a stable RLOF (with $P_{\mathrm{i}} \sim 10-1000$ d) to form a WD binary, and the secondary of the WD binary needs to fill its Roche lobe when its helium core mass is in an appropriate range (see Fig. 1 of Han et al. 2002). The ejection of the resultant CE leads to a close WD+EHB binary. As the WD can spiral in deeper in the envelope during the $\mathrm{CE}$ ejection, the WD+EHB can have a much shorter orbital period (as short as $\sim 0.02 \mathrm{~d}$ for a small $t$ ) than the MS+EHB binary from the first CE ejection channel. For a large $t$, the WD spirals in the envelope of a less massive secondary (the envelope is more loosely bound), and the resultant
Table 1. Parameters for simulation sets.

\begin{tabular}{ccccc}
\hline \hline Set & $n\left(q^{\prime}\right)$ & $q_{\text {crit }}$ & $\alpha_{\mathrm{CE}}$ & $\alpha_{\text {th }}$ \\
\hline 1 & constant & 1.5 & 0.75 & 0.75 \\
2 & constant & 1.5 & 0.50 & 0.50 \\
3 & constant & 1.5 & 1.0 & 1.0 \\
4 & constant & 1.2 & 0.75 & 0.75 \\
5 & uncorrelated & 1.5 & 0.75 & 0.75 \\
\hline
\end{tabular}

WD+EHB binary is wider. For the merger channel, a binary system first needs to experience a stable RLOF (with $P_{\mathrm{i}} \sim 4-250 \mathrm{~d}$ and $M_{1 \mathrm{i}} \sim 0.95-2 M_{\odot}$ ) to produce a helium WD binary, and the binary experiences a $\mathrm{CE}$ evolution to form a helium WD pair. The pair may coalesce to form a single EHB star due to angular momentum loss of gravitational wave radiation. The EHB star produced from this channel has a wider mass range $\left(0.4-0.8 M_{\odot}\right)$ than from other channels. The mass range is smaller for a small $t\left(0.56-0.64 M_{\odot}\right.$ for $\left.t=2 \mathrm{Gyr}\right)$ as only very close helium WD pairs have time enough to merge, but the range becomes larger with a large $t\left(\sim 0.4-0.8 M_{\odot}\right.$ for $\left.t=15 \mathrm{Gyr}\right)$.

In the process of $\mathrm{CE}$ ejection, the orbital energy released by the orbital decay of the embedded binary is used to overcome the binding energy of the CE. As is usual, I defined two parameters: the $\mathrm{CE}$ ejection efficiency $\alpha_{\mathrm{CE}}$, i.e. the fraction of the released orbital energy used to overcome the binding energy; and $\alpha_{\text {th }}$, which defines the fraction of the thermal energy contributing to the binding energy of the CE. As in the HPMM model, I adopted $q_{\text {crit }}=1.5$ (for stable RLOF on the first giant branch), and $\alpha_{\mathrm{CE}}=\alpha_{\mathrm{th}}=0.75$ as the best choices, and varied them to see their effects.

To obtain the distributions of properties of EHB stars at different ages, I have performed detailed Monte Carlo simulations with the binary population synthesis code developed for the HPMM model. In the simulation, I followed the evolution of 10 million sample binaries according to grids of stellar models of solar metallicity and the evolution channels leading to EHB stars. I adopted the following input for the simulations (see Han et al. 1995):

1. a single star burst is adopted (note that the star-formation rate is taken to be constant over the last $15 \mathrm{Gyr}$ in the HPMM model);

2. the initial mass function of Miller \& Scalo (1979) is adopted;

3. for the initial mass ratio distribution $n\left(q^{\prime}\right)$ (where $q^{\prime}$ is the ratio of secondary to primary), I adopted (a) a constant mass ratio distribution, or alternatively (b) a mass ratio distribution where the masses are uncorrelated and drawn independently from a Miller \& Scalo (1979) initial mass function;

4. I take the distribution of separations to be constant in $\log a$ for wide binaries, where $a$ is the orbital separation. The adopted distribution implies that $\sim 50 \%$ of stellar systems are binary systems with orbital periods less than $100 \mathrm{yr}$.

Similar to the HPMM model, I have carried out 5 sets of Monte Carlo simulations altogether for Population I by varying the model parameters over a reasonable range (see Table 1). As in the HPMM model, the standard simulation set (set 1) has a constant initial mass ratio distribution, $q_{\text {crit }}=1.5$, and $\alpha_{\mathrm{CE}}=\alpha_{\mathrm{th}}=0.75$.

\section{Results}

The simulations give EHB stars at various stellar population ages. In order to see the importance of individual evolution channels leading to the formation of EHB stars, I plotted in Fig. 1 the 


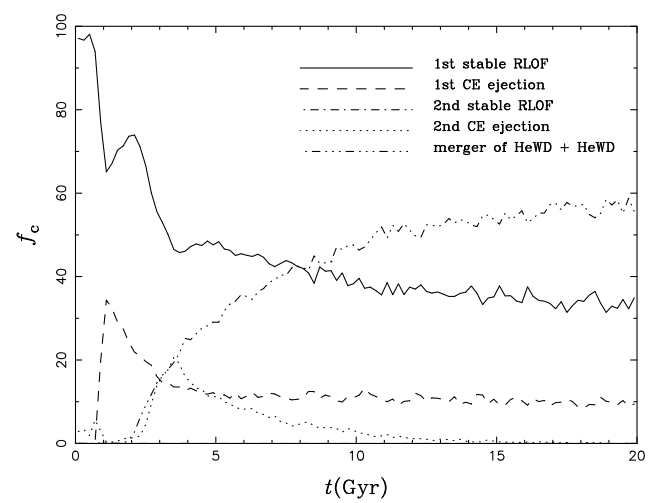

Fig. 1. The evolution of the fraction $f_{\mathrm{c}}$ (in percentage) of EHB stars originated from each channel for the standard simulation set (set 1), i.e., the 1st stable RLOF channel (wide EHB+MS binaries); the 1st $\mathrm{CE}$ ejection channel (close EHB+MS binaries); the 2nd stable RLOF channel (wide EHB+WD binaries); the 2nd CE ejection channel (close $\mathrm{EHB}+\mathrm{WD}$ binaries); and the merger channel (single EHB stars). No selection effect has been applied to the lines. Note that there are no EHB binaries from the 2nd stable RLOF channel.

fractions of EHB stars from different channels (the 1st stable RLOF channel for wide EHB+MS binaries; the 1st CE ejection channel for close EHB+MS binaries; the 2nd stable RLOF channel for wide $\mathrm{EHB}+\mathrm{WD}$ binaries; the 2nd $\mathrm{CE}$ ejection channel for close $\mathrm{EHB}+\mathrm{WD}$ binaries; and the merger channel for single EHB stars) at stellar population age $t$ for the standard simulation set (set 1).

Figure 2 shows the evolution of the distribution of orbital periods of EHB binaries with stellar population age $t$ for the standard simulation set (set 1). The EHB binaries with orbital periods $P<5 \mathrm{~d}$ can be detected observationally in a GC (Moni Bidin et al. 2008), and I, therefore, showed, in Fig. 3, the fractions of the binaries among all the EHB stars, including both binaries and singles, at stellar population age $t$. In order to see how the fractions can be affected by model parameters, Fig. 3 also displays other simulation sets with various model parameters. In the figure, I have applied the GK selection effect, which is the selection against EHB stars with companions of spectral type $G$ and $\mathrm{K}$ (usually MS stars), and is the most important selection effect in observations of EHB stars ${ }^{1}$.

\section{Discussion}

Figure 1 shows the fraction of EHB stars originated from each channel. We see that the 1 st stable RLOF is dominant for $t<$ 8 Gyr. The resultant wide MS+EHB binaries, however, may not be observed due to the GK selection effect as the MS secondaries are too massive and bright. The 1 st $\mathrm{CE}$ ejection channel and the 2nd CE ejection channel have a contribution peak at $t \sim 1.2 \mathrm{Gyr}$ and $t \sim 3.5 \mathrm{Gyr}$, respectively. The merger channel starts to contribute noticeably at $t \sim 2 \mathrm{Gyr}$ and dominates at $t \gtrsim 8 \mathrm{Gyr}$, or at $t \gtrsim 3.5 \mathrm{Gyr}$ if the GK selection is considered.

Figure 2 shows the distributions of orbital periods of EHB binaries at various ages of a stellar population. For $t=0.5 \mathrm{Gyr}$, the distribution has 3 peaks. The left peak (the small one at $\log (P / \mathrm{d}) \sim-1.3)$ is from the 2 nd CE ejection channel, where a WD has spiralled in deeply into a tightly bound envelope of its companion (with $M_{2 \mathrm{i}}>2 M_{\odot}$ ) to form a close WD+EHB

${ }^{1}$ We applied the GK selection effect in the following way. If an EHB binary has a MS companion and the effective temperature of the companion is above $4000 \mathrm{~K}$ or the companion is brighter than the EHB star, the system is excluded. See the HPMM model for details.

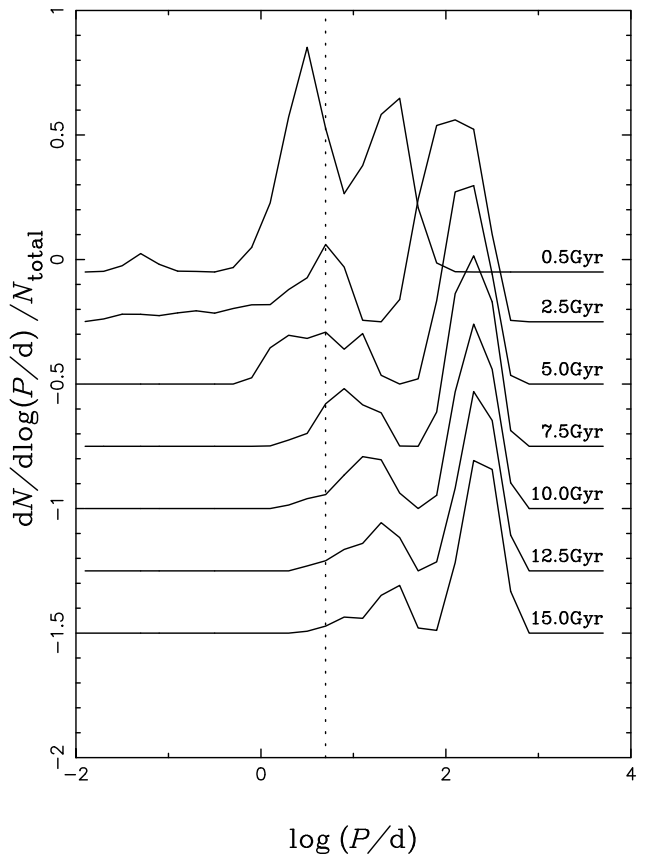

Fig. 2. The evolution of the distributions of orbital periods with stellar population age $t$ for the standard simulation set (set 1). $P$ is orbital period, and $N_{\text {total }}$ is the total number of EHB stars, including both binaries and singles. The stellar population ages are denoted and each distribution has been applied an offset of $-0.1 \times(t / \mathrm{Gyr})$, where $t$ is the age. The distributions are without any selection effect. If we apply the GK selection effect, the far-right peaks, which are mainly EHB binaries with more massive MS companions from the 1st stable RLOF channel, disappear. The vertical dotted line denotes $P=5 \mathrm{~d}$.

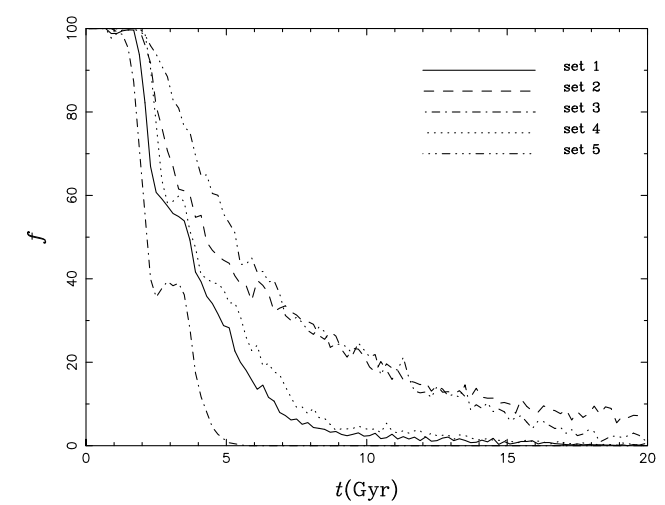

Fig. 3. The evolution of the fraction $f$ (in percentage) of close EHB binaries (with orbital periods $P<5 \mathrm{~d}$ ) among all EHB stars, including both binaries and singles. Note that the GK selection effect is applied. If the selection effect is not considered, $f$ would be smaller due to the fact that the GK selection effect excludes wide EHB+MS binaries from the first stable RLOF channel.

binary. Both the middle peak and the right peak are from the 1st stable RLOF channel with $M_{1 \mathrm{i}}>2 M_{\odot}$, while the middle peak is from stable RLOF on the Hertzsprung gap (with a mass ratio of $\left.q_{\mathrm{i}} \sim 1-3\right)$ and the right peak from stable RLOF on the first giant branch (with a mass ratio of $q_{\mathrm{i}} \sim 1-1.5$ ). For $t=2.5 \mathrm{Gyr}$, the distribution has two peaks. The left peak is from the $1 \mathrm{st}$ CE ejection channel and the part to the left of the peak is from the 2nd CE ejection channel. The right peak is from the 1st stable RLOF channel, and the orbital period corresponding to the right peak is significantly larger than that at $t=0.5 \mathrm{Gyr}$, which is due to the fact that stellar radius at the tip of the first giant branch is significantly larger for $M_{1 \mathrm{i}}<2 M_{\odot}$ than for $M_{1 \mathrm{i}}>2 M_{\odot}$. 
For $t=5 \mathrm{Gyr}$ or larger $t$, the 2 nd CE ejection channel produces WD+EHB binaries with orbital periods similar to that of MS+EHB binaries from the 1st CE ejection channel, as the mass donors (the primary for the 1 st $\mathrm{CE}$ ejection channel, or the secondary for the 2nd CE ejection channel) are less massive than $2 M_{\odot}$, and the envelopes are similarly loosely bound near the tip of the first giant branch. For a large $t$, the radius (and the corresponding $P_{\mathrm{i}}$ ) at the tip of the first giant branch is bigger for the donor, and the envelope is also more loosely bound, and therefore the left peak moves toward a longer orbital period for a large $t$.

The EHB binaries with orbital periods $P<5 \mathrm{~d}$ are from the 1 st CE ejection channel or the 2 nd CE ejection channel. Figure 3 shows the evolution of the fraction $f$ of close EHB binaries (with $P<5 \mathrm{~d}$ ) with stellar population age $t$. We see that $f$ decreases sharply with $t$, which is largely due to the increasing importance of the merger channel with $t$ (see Fig. 1). For the standard simulation set (set 1 ), $f$ is $\sim 2.5 \%$ for $t=10 \mathrm{Gyr}$. In order to see the effects of model parameters, I performed 5 sets of simulations altogether. Set 2 adopted smaller common envelope ejection efficiency and the orbital periods of close EHB binaries are, therefore, smaller, leading to bigger $f$ for $t>1$ Gyr. Set 3, on the other hand, adopted bigger efficiency and, therefore, $f$ is smaller. Set 4 adopted smaller $q_{\mathrm{c}}$ for stable RLOF on the first giant branch, and this makes CE more likely, and leads to more EHB binaries from CE ejection channels, which results in bigger $f$. Set 5 adopted an uncorrelated initial mass ratio distribution, which means a large initial mass ratio $q_{\mathrm{i}}$ is more likely and as a consequence $\mathrm{CE}$ is more likely experienced and, therefore, $f$ is larger. Note that the parameters adopted for the standard simulation set (set 1) produce good matches between observation and theory for field EHB stars (see HPMM for details).

There is a very high fraction of close EHB binaries in the field ( 2/3, see Maxted et al. 2001), but there is a lack of close EHB binaries in GCs. Moni Bidin et al. (2006) and Moni Bidin et al. (2008) found that the binary fraction $f$ (for $P<5 \mathrm{~d}$ ) is very small in NGC 6752, with a most likely value of $f=4 \%$ and an upper limit of $f=16 \%$ at the $95 \%$ confidence level. They speculated that the sharp contrast between field EHB stars and GC EHB stars may be due to a possible $f-t$ relation $^{2}$. They analysed the HPMM model and Fig. 7 of Han et al. (2007) and found that such a relation is possible ${ }^{3}$. This Letter showed that the $f-t$ relation clearly exists, in support of the speculation of Moni Bidin et al. (2008). For field EHB stars, their progenitors have a wide spectrum of masses due to the continuous star formation rate during the past, and young (massive) progenitors are more likely to produce closer EHB binaries, and therefore the binary fraction is much higher (see the HPMM model).

Gratton et al. (2003, 2003) derived the average metallicity and the age of NGC 6752 to be $[\mathrm{Fe} / \mathrm{H}]=-1.48 \pm 0.07$ and $13.8 \pm 1.1 \mathrm{Gyr}$, respectively. The standard simulation set (set 1) gives the fraction of close EHB binaries with $P<5 \mathrm{~d}$ to be $f \sim 1.3 \%$ at $t=13.8 \mathrm{Gyr}$, close to the fraction of $4 \%$ observed by Moni Bidin et al.(2008) if we consider that $f$ is very sensitive to the model parameters, e.g. CE ejection efficiency, in each simulation set (see Fig. 3). Note the parameters adopted for the

\footnotetext{
${ }^{2}$ Napiwotzski et al. (2004) invoked a possible $f-t$ or $f-[\mathrm{Fe} / \mathrm{H}]$ relation to explain the binary fraction difference between their survey for EHB stars and previous surveys for field EHB stars. Their sample contains more thick disk or halo stars.

${ }^{3}$ Moni Bidin et al. (2008) also argued that dynamical effects may not contribute to the formation of EHB stars in GCs, as there is a lack of radial gradients among EHB stars in NGC 2808 (Bedin et al. 2000) and $\omega$ Centauri (D'Cruz et al. 2000).
}

standard simulation set give a high binary frequency among field EHB stars ( $\sim 55 \%$ after selection effects are taken, see Table 2 of Han et al. 2003), consistent with observations of field EHB stars. Note also that our model is for solar metallicity. For low metallicity, a star has a more tightly-bound envelope. Therefore, the EHB binaries from CE ejections have shorter orbital periods, and the helium WD pairs would be closer and should be more likely to merge to form single EHB stars. However, the production of EHB stars are not expected to be affected much by metallicity in the HPMM binary model.

The CE ejection efficiency strongly affects the distribution of close EHB binaries (see Fig. 3). If we can obtain the parameters, i.e., the orbital period, the mass of the EHB star, and the mass of the companion, of a close EHB binary in a GC of known age and metallicity, we may constrain the $\mathrm{CE}$ ejection efficiency. From the age and metallicity of a GC, we can infer the mass of the progenitor of the EHB star. From the mass of the EHB star and the mass of the companion, we would get the location of the $\mathrm{CE}$ on the evolutionary track of the progenitor, and we would then know the binding energy of the CE and the separation before the $\mathrm{CE}$. The separation after the $\mathrm{CE}$ can be easily calculated from the binary parameters observed. Then it would be straight forward to derive the CE ejection efficiency. I, therefore, propose here that precise determination of the physical parameters of close EHB binaries in GCs would possibly give the strictest constraint on CE ejection efficiency. However, such an approach seems to be too remote. A more realistic observational test of the results in this Letter would be searching for EHB binaries with orbtial periods of $\sim 10-20 \mathrm{~d}$ in a GC (see Fig. 2).

Acknowledgements. I thank an anonymous referee for his/her generous comments which helped to improve the paper, and Ph. Podsiadlowski for stimulating discussions. This work was in part supported by the Natural Science Foundation of China under Grant Nos 10433030, 10521001 and 2007CB815406.

\section{References}

Bedin, L. R., Piotto, G., Zoccali, M., et al. 2000, A\&A, 363, 159 Bressan, A., Chiosi, C., \& Fagotto, F. 1994, ApJS, 94, 63

D’Cruz, N. L., Dorman, B., Rood, R. T., \& O'Connell, R. W. 1996, ApJ, 466, 359

D’Cruz, N. L., O'Connell, R. W., Rood, R. T., et al. 2000, ApJ, 530, 352

Gratton, R. G., Bragaglia, A., Carretta, E., et al. 2003, A\&A, 408, 529

Gratton, R. G., Bragaglia, A., Carretta, E., et al. 2005, A\&A, 440, 901

Green, R. F., Schmidt, M., \& Liebert, J. 1986, ApJS, 61, 305

Han, Z., Podsiadlowski, Ph., \& Eggleton, P. P. 1995, MNRAS, 272, 800

Han, Z., Podsiadlowski, Ph., Maxted, P. F. L., Marsh, T. R., \& Ivanova, N. 2002, MNRAS, 336, 449

Han, Z., Podsiadlowski, Ph., Maxted, P. F. L., \& Marsh, T. R. 2003, MNRAS, 341,669

Han, Z., Podsiadlowski, Ph., \& Lynas-Gray, A. E. 2007, MNRAS, 380, 1098

Heber, U. 1986, A\&A, 155, 33

Kilkenny, D., Koen, C., O’Donoghue, D., \& Stobie, R. S. 1997, MNRAS, 285, 640

Lee, Y. W. 1994, ApJ, 430, L113

Maxted, P. F. L., Heber, U., Marsh, T. R., \& North, R. C. 2001, MNRAS, 326, 1391

Mengel, J. G., Norris, J., \& Gross, P. G. 1976, ApJ, 204, 488

Miller, G. E., \& Scalo, J. M. 1979, ApJS, 41, 513

Moni Bidin, C., Moehler, S., Piotto, G., et al. 2006, A\&A, 451, 499

Moni Bidin, C., Catelan, M., \& Altmann, M. 2008, A\&A, 480, L1

Napiwotzki, R., Carl, C. A., Lisker, T., et al. 2004, Ap\&SS, 291, 321

O’Tool, S. J., Heber, U., \& Benjamin, R. A. 2004, A\&A, 422, 1053

Paczyński, B. 1976, Common Envelope Binaries, in Structure and Evolution

of Close Binaries, ed. P. P. Eggleton, S. Mitton, \& J. Whelan (Dordrecht: Kluwer), 75

Saffer, R. A., Bergeron, P., Koester, D., \& Liebert, J. 1994, ApJ, 432, 351

Sweigart, A. V. 1997, ApJ, 474, L23

Tutukov, A. V., \& Yungelson, L. R. 1990, A. Zh., 67, 109

Webbink, R. F. 1984, ApJ, 277, 355

Yi, S. K., Demarque, P., \& Oemler, A. Jr. 1997, ApJ, 486, 201 